\title{
Concurrent validity of kidney transplant questionnaire in US renal transplant recipients
}

This article was published in the following Dove Press journal:

Patient Preference and Adherence

12 October 2011

Number of times this article has been viewed

\author{
Marie A Chisholm-Burns ${ }^{1,2}$ \\ Steven R Erickson ${ }^{3}$ \\ Christina A Spivey' \\ Rainer WG Gruessner ${ }^{2}$ \\ Bruce Kaplan ${ }^{4}$ \\ 'Department of Pharmacy Practice \\ and Science, University of Arizona \\ College of Pharmacy, Tucson, AZ; \\ ${ }^{2}$ Department of Surgery, University of \\ Arizona College of Medicine, Tucson, \\ AZ; ${ }^{3}$ Department of Clinical Sciences, \\ University of Michigan College of \\ Pharmacy, Ann Arbor, MI; ${ }^{4}$ Department \\ of Medicine, The University of Arizona \\ College of Medicine Tucson, AZ, USA
}

Correspondence: Marie A Chisholm-Burns The University of Arizona College of Pharmacy, 1295 N Martin Ave, PO Box 210202, Tucson,

AZ 85721 , USA

Tel +I 5206262298

Fax+I 5206267355

Email chisholm@pharmacy.arizona.edu
Background: Valid instrumentation in the assessment of health-related quality of life (HQoL) in renal transplant recipients is critical to identifying particular nuances and determinants of $\mathrm{HQOL}$ in this population. Therefore, the validity of disease-specific instruments to measure HQoL in renal transplant recipients, such as the Kidney Transplant Questionnaire (KTQ), needs further investigation. The objective of this study was to assess the concurrent validity of the KTQ in adult US renal transplant recipients using the well established SF-12 Health Survey version 2 (SF-12v2) as the comparison instrument.

Methods: One hundred and fourteen renal transplant recipients met the following inclusion criteria for this study, ie, were at least 21 years of age, more than two years post-transplant, and receiving immunosuppressant therapy. Subjects were asked to complete a series of HQoL instruments, ie, the KTQ and the SF-12v2 (physical component summary [PCS-12] and mental component summary [MCS-12]). Descriptive statistics were calculated, and correlational analyses were conducted to examine the concurrent validity of the HQoL instruments.

Results: Among 100 participants ( $87.7 \%$ response rate), the majority of participants were male (52\%), had deceased donor transplants (63\%), and received Medicare benefits (84\%). PCS-12 was positively correlated with three of five KTQ subscales $(P<0.05)$, ie, KTQ-physical $(r=0.43)$, KTQ-fatigue $(r=0.42)$, and KTQ-uncertainty/fear $(r=0.2)$. MCS-12 was positively correlated with all KTQ subscales $(P<0.01)$, ie, KTQ-physical $(r=0.26)$, KTQ-fatigue $(r=0.48)$, KTQuncertainty/fear $(r=0.33)$, KTQ-emotional $(r=0.47)$, and KTQ-appearance $(r=0.28)$.

Conclusion: The findings support the concurrent validity of the KTQ in US renal transplant recipients. Future studies should continue exploring the validity of the KTQ, as well as its practical and research utility in HQoL measurement in the renal transplant population.

Keywords: concurrent validity, kidney transplant questionnaire, quality of life, renal transplant recipients, SF-12 Health Survey

\section{Introduction}

The purpose of renal transplant is not simply to extend life and improve health outcomes, but to enhance quality of life. Health-related quality of life (HQoL) is defined by the World Health Organization as “... individuals' perception of their position in life in the context of the culture and value systems in which they live and in relation to their goals, expectations, standards, and concerns". ${ }^{1}$ HQoL is an important outcome measure in the renal transplant field, and its spectrum includes physical, psychological, social, and general well-being. Rebollo et $\mathrm{al}^{2}$ described the necessity of examining HQoL in transplant and other patient populations as follows to:

- Evaluate efficacy of medical interventions

- Improve the clinical decision-making process 
- Assess quality of care

- Estimate patient population's health care needs

- Better understand causes and consequences of health differences within a patient population

Research demonstrates that HQoL tends to improve after undergoing renal transplant as compared with remaining on dialysis. ${ }^{3-5}$ However, HQoL changes in renal transplant recipients are variable, and may be influenced by clinical, social, demographic, and environmental factors. ${ }^{4}$ Because of this noted variation, valid, reliable, and well constructed instrumentation in the assessment of HQoL is critical to identifying particular nuances or determinants of HQoL in renal transplant recipients. To that end, a small number of HQoL instruments specific to renal transplantation have been developed. These disease-specific instruments tend to focus on those symptoms, effects, and issues that are commonly associated with renal transplantation. One such instrument is the Kidney Transplant Questionnaire (KTQ), developed by Laupacis et al, which assesses characteristics and conditions associated with life post-transplant. ${ }^{6}$ Although previous studies provide evidence of the validity of the KTQ in non-US renal transplant populations, a literature search (PubMed, years unlimited) revealed little to no evidence regarding the validity of the instrument among US renal transplant recipients. ${ }^{2,6,7}$ Because there are few $\mathrm{HQoL}$ instruments available specific to the renal transplant population, it is important to develop support for the validity of existing measures such as the KTQ. Access to valid, disease-specific HQoL measures may allow for more complete comprehension of the quality of life-related benefits (ie, improvements in HQoL in certain domains) and limits (ie, circumstances under which HQoL does not improve or improves only minimally) of transplant as a medical intervention in US renal transplant recipients. Therefore, the objective of this study was to assess the concurrent validity (defined as evaluation of an instrument against an already validated measure or criterion of the construct the instrument should be assessing ${ }^{8-10}$ ) of the KTQ in adult US renal transplant recipients using the well established SF-12 Health Survey version 2 (SF-12v2) as the comparison instrument.

\section{Materials and methods}

One hundred and fourteen renal transplant recipients met the following enrollment criteria for this crosssectional survey (note that recruitment of renal transplant recipients, and therefore inclusion criteria, was part of a larger, ongoing randomized controlled trial conducted by Chisholm-Burns et al) and were: at least 21 years of age; more than two years post-transplant; and receiving immunosuppressant therapy from the Apothecary Shops (a chain of pharmacies based in the Southwest US). As part of enrollment into the randomized controlled trial, participants were asked to complete and return mailed surveys, which included a demographics questionnaire and the KTQ and SF-12v2 HQoL instruments. Renal transplant recipients who did not return the survey packet within two weeks received one follow-up telephone call reminder. This study was approved by the Institutional Review Board of The University of Arizona.

\section{HQoL instrumentation}

\section{Kidney Transplant Questionnaire}

The KTQ is a 25 -item questionnaire that includes five domains or subscales, ie, physical symptoms (based on six items), fatigue (based on five items), uncertainty/fear (based on four items), appearance (based on four items), and emotional (based on six items). ${ }^{6} \mathrm{~A}$ mean score ranging from 1 to 7 is reported for each of the five subscales, with higher scores representing better functioning, well-being, or fewer problems. Evidence supporting the validity and reliability (Cronbach's alpha and intraclass coefficients of subscales range from 0.69 to 0.90 ) of the KTQ has been reported in non-US renal transplant recipients. ${ }^{2,6,7}$

\section{SF-I 2 Health Survey version 2}

The SF-12v2, a well validated brief, generic HQoL questionnaire, is a shortened form of the SF-36 Health Survey (SF-36). ${ }^{11}$ The SF-36 is a commonly used HQoL measure in the renal transplant literature, and has been utilized to validate other disease-specific symptom and health status measures. ${ }^{3,12-14}$ Similar to the SF-36, the SF-12v2 assesses functional status, well-being, and perceptions of health status across eight domains, ie, physical functioning, role functioning-physical, bodily pain, general health, vitality, social functioning, role functioning-emotional, and mental health. The scores from the eight domains are used to calculate a physical component summary (PCS-12) score and a mental component summary (MCS-12) score. Note, for studies such as ours in which sample sizes are relatively small (ie, $<500$ participants), reporting of just the PCS-12 and MCS-12 scores is appropriate; thus, we utilized only the PCS-12 and MCS-12 scores in our concurrent validity analysis. ${ }^{11}$ The domain scores derived from the SF-12v2 are based on fewer items and fewer defined levels of health than the SF-36 and, as a result, are estimated with less precision and less reliability. Despite these possible limitations, the 
SF-12v2 was selected because its brevity is appealing in reducing burden on patients, particularly when administered in conjunction with a lengthier instrument such as the KTQ. Scores on the SF-12v2 are based on normative scaling and range from 0 to 100 , with 50 considered the average functioning score. Higher PCS-12 and MCS-12 scores are indicative of better functioning and well-being. Across numerous patient populations, Cronbach's alpha coefficients for the PCS-12 and MCS-12 greater than 0.70 have been reported, and evidence supporting the validity of the SF-12v2 has been found. ${ }^{11}$

\section{Hypotheses}

The KTQ has not been validated using the SF-12v2, but Rebollo et $\mathrm{al}^{2}$ have previously validated the KTQ in Spanish renal transplant recipients using the SF-36, which has physical and mental component scores similar to those of the PCS-12 and MCS-12 of the SF-12v2. ${ }^{2}$ The following exploratory hypotheses are based on the correlational findings of Rebollo et al:

- The PCS-12 will be positively correlated at the low to moderate level ( $r=0.2-0.4$ ) with the KTQ-physical, KTQ-fatigue, and KTQ-appearance subscales, and will be positively correlated at the low level $(r=0.1-0.2)$ with the KTQ-uncertainty/fear subscale.

- The MCS-12 will be positively correlated at the greater than moderate level $(r>0.4)$ with the KTQ-emotional, KTQ-uncertainty/fear, and KTQ-fatigue subscales, and will also be positively correlated at the low to moderate level ( $r=0.2-0.4)$ with the KTQ-physical and KTQappearance subscales.

\section{Statistical analysis}

To perform the statistical analyses, we used SPSS version 18/PASW Statistics 18 (SPSS Inc, Chicago, IL). Descriptive statistics of demographic and HQoL variables were calculated. To assess reliability, Cronbach's alpha coefficients were calculated for the HQoL instruments. Ceiling and floor effects for each scale were tested by running frequencies for each scale and documenting the percentage of respondents who scored at the top and bottom of each scale. Ceiling and floor effects less than 15\% were considered minimal, while effects greater than $15 \%$ were considered moderate to substantial, according to criteria used by McHorney and Tarlov. ${ }^{15}$ To assess concurrent validity, analyses were conducted to examine Pearson's $r$ correlations among the PCS-12, MCS-12, and KTQ subscales. An alpha priori level of 0.05 was used.

\section{Results}

The response rate was $87.7 \%$ (100 of 114 renal transplant recipients). The mean age of respondents was $51.0 \pm 13.2$ years and the mean months post-transplant was $39.7 \pm 29.8$ (Table 1$)$. The majority of participants were male $(52 \%)$, had deceased donor transplants (63\%), and received Medicare benefits ( $84 \%$, Table 1). The mean scores on the HQoL instruments were as follows: PCS-12, $43.7 \pm 11.6$;

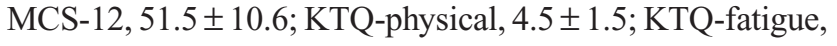
$5.4 \pm 1.4$; KTQ-uncertainty/fear, $5.4 \pm 1.3$; KTQ-appearance, $6.8 \pm 0.6$; and KTQ-emotional, $5.4 \pm$ 1.2. Cronbach's alpha for each scale is presented in Table 2. Neither ceiling nor floor effects were noted for the PCS-12 or MCS-12. There were modest ceiling effects for KTQ-physical, KTQ-fatigue, KTQ-uncertainty/fear, and KTQ-emotional subscales, ranging from $15 \%$ to $27 \%$ of respondents receiving the highest possible score. A substantial ceiling effect was present for the KTQ-appearance subscale, with $84 \%$ of renal transplant recipients receiving the highest possible score.

The correlation matrix is presented in Table 3. Generally, the following patterns of correlations between the HQoL

Table I Demographic characteristics $(n=100)$

\begin{tabular}{|c|c|}
\hline Characteristic & Frequency (\%) \\
\hline Mean age $\pm S D$, years & $51.0 \pm 13.2$ \\
\hline \multicolumn{2}{|l|}{ Gender } \\
\hline Male & $52(52)$ \\
\hline Female & $48(48)$ \\
\hline \multicolumn{2}{|l|}{ Education } \\
\hline Less than high school & $17(17)$ \\
\hline High school graduate & $23(23)$ \\
\hline Some college/associate degree & $35(35)$ \\
\hline College graduate or higher & $20(20)$ \\
\hline Other & $2(2)$ \\
\hline Missing data & $3(3)$ \\
\hline \multicolumn{2}{|l|}{ Employment status } \\
\hline Employed & $33(33)$ \\
\hline Not employed & $65(65)$ \\
\hline Missing data & $2(2)$ \\
\hline \multicolumn{2}{|l|}{ Marital status } \\
\hline Not married & $59(59)$ \\
\hline Married & $4 I(4 I)$ \\
\hline \multicolumn{2}{|l|}{ Race } \\
\hline White & $50(50)$ \\
\hline Other & $50(50)$ \\
\hline \multicolumn{2}{|l|}{ Received Medicare benefits } \\
\hline No & $16(16)$ \\
\hline Yes & $84(84)$ \\
\hline \multicolumn{2}{|l|}{ Type of transplant } \\
\hline Living donor & $37(37)$ \\
\hline Deceased donor & $63(63)$ \\
\hline Mean months post-transplant $\pm \mathrm{SD}$ & $39.7 \pm 29.8$ \\
\hline
\end{tabular}

Abbreviation: SD, standard deviation. 
Table 2 Cronbach's alpha for the health-related quality of life instruments

\begin{tabular}{ll}
\hline HQoL instrument & Cronbach's alpha \\
\hline PCS-I2 & 0.92 \\
MCS-I2 & 0.88 \\
KTQ-physical & 0.82 \\
KTQ-fatigue & 0.90 \\
KTQ-uncertainty/fear & 0.63 \\
KTQ-appearance & 0.62 \\
KTQ-emotional & 0.82 \\
\hline
\end{tabular}

Abbreviations: HQoL, health-related quality of life; KTQ, Kidney Transplant Questionnaire; MCS-12, mental component summary of the SF-12 health survey; PCS-12, physical component summary of the SF-12 health survey.

scores were confirmed. As hypothesized, the PCS-12 was positively correlated at the low level $(r=0.2 ; P<0.05)$ with the KTQ-uncertainty/fear subscale. Correlations between the PCS-12 and the KTQ-physical and KTQ-fatigue subscales were slightly higher than hypothesized $(r>0.4 ; P<0.001)$. Although hypothesized to be positively correlated at the low level, the PCS-12 and KTQ-appearance subscale were not significantly correlated $(P>0.05)$. Also as hypothesized, the MCS-12 was positively correlated at the greater than moderate level with the KTQ-emotional and KTQ-fatigue subscales $(r>0.4 ; P<0.001)$, and was positively correlated at the low to moderate level with the KTQ-physical and KTQ-appearance subscales ( $r=0.26$ and 0.28 , respectively; $P<0.01)$. Although hypothesized to be positively correlated at the greater than moderate level, the MCS-12 was correlated at the low to moderate level with the KTQ-uncertainty/fear subscale $(r=0.33 ; P<0.01)$.

\section{Discussion}

As there are few instruments designed specifically to assess HQoL in the renal transplant population, comprehensive validity testing of existing measures such as the KTQ is needed across renal transplant populations. The current study is the first to examine the concurrent validity of the
KTQ in a sample of US renal transplant recipients using the SF-12v2 as the comparison instrument. The findings of our study provide support for the reliability of both instruments. We found acceptable to good Cronbach's alphas $(>0.75)$ for five of seven scales (PCS-12, MCS-12, KTQ-physical, KTQfatigue, and KTQ-emotional). ${ }^{16}$ The two remaining scales, the KTQ-appearance and KTQ-uncertainty/fear, had Cronbach's alphas approaching acceptability $(>0.6) .{ }^{16}$ Reliability coefficients found in this study for each instrument are similar to those noted in previous studies. , $^{2,11,17}$

Study findings provide support for the concurrent validity of the KTQ in our sample, because the positive correlations between the KTQ and SF-12v2 indicate that the scales hypothesized to measure similar concepts were, for the most part, significantly associated. In examining the relationship between the KTQ and SF-12v2, correlations which are clinically and psychometrically sensible were found between the KTQ subscale scores and SF-12v2 component summary scores. To elaborate, the PCS-12 is most highly correlated with the KTQ-physical ( $r=0.43)$ and KTQ-fatigue subscales $(r=0.42)$, while the MCS-12 is most highly correlated with the KTQ-emotional ( $r=0.47)$ and KTQ-fatigue $(r=0.48)$ subscales. The findings regarding the fatigue subscale are particularly important given that the causes and effects of fatigue have both physical and mental elements. Our results offer the first body of evidence concerning the concurrent validity of the KTQ with the SF-12v2.

The findings regarding the relationships between the KTQ subscales and the SF-12v2 vary somewhat from those presented by Rebollo et al in their examination of the validity of the KTQ using the SF-36 in a sample of Spanish renal transplant recipients. ${ }^{2}$ For example, Rebollo et al found that correlations between the KTQ subscales and the physical component summary of the SF-36 (PCS-36) were low (less than 0.3 for all KTQ subscales), while two of the correlations found between the PCS-12 and KTQ

Table 3 Health-related quality of life instrument correlation matrix

\begin{tabular}{|c|c|c|c|c|c|c|c|}
\hline Instrument & PCS- 12 & MCS-I 2 & $\begin{array}{l}\text { KTQ- } \\
\text { physical }\end{array}$ & $\begin{array}{l}\text { KTQ- } \\
\text { fatigue }\end{array}$ & $\begin{array}{l}\text { KTQ- } \\
\text { uncertainty/fear }\end{array}$ & $\begin{array}{l}\text { KTQ- } \\
\text { appearance }\end{array}$ & $\begin{array}{l}\text { KTQ- } \\
\text { emotional }\end{array}$ \\
\hline PCS-12 & - & 0.112 & $0.43 * * *$ & $0.42 * * *$ & $0.20 *$ & -0.01 & 0.10 \\
\hline MCS- 12 & 0.112 & - & $0.26 * *$ & $0.48 * * *$ & $0.33 * *$ & $0.28 * *$ & $0.47 * * *$ \\
\hline KTQ-physical & $0.43 * * *$ & $0.26 * *$ & - & $0.59 * * *$ & $0.47 * * *$ & $0.29 * *$ & $0.52 * * *$ \\
\hline KTQ-fatigue & $0.42 * * *$ & $0.48 * * *$ & $0.59 * * *$ & - & $0.54 * * *$ & $0.31 * *$ & $0.61 * * *$ \\
\hline KTQ-uncertainty/fear & $0.20 *$ & $0.33^{* *}$ & $0.47 * * *$ & $0.54 * * *$ & - & $0.40 * * *$ & $0.7 \mid * * *$ \\
\hline KTQ-appearance & -0.01 & $0.28 * *$ & $0.29 * *$ & $0.31 * *$ & $0.40 * * *$ & - & $0.36 * * *$ \\
\hline KTQ-emotional & 0.10 & $0.47 * * *$ & $0.52 * * *$ & $0.6 I * * *$ & $0.7 \mid * * *$ & $0.36 * * *$ & - \\
\hline
\end{tabular}

Notes: $* P<0.05$; $* * P<0.01$; $* * * P<0.001$.

Abbreviations: KTQ, Kidney Transplant Questionnaire; MCS-12, mental component summary of the SF-12 health survey; PCS-12, physical component summary of the SF-I 2 health survey. 
subscales in this study were greater than $0.4 .^{2}$ Additionally, although the correlations found in the current study using the MCS-12 were significant, Rebollo et al found correlations between the KTQ subscales and the mental component summary of the SF-36 (MCS-36) that were considerably higher than those found in our study. ${ }^{2}$ This is particularly noteworthy in the case of KTQ-uncertainty/fear. We speculate that differences in time post-transplant may be associated with the variations in results found between the current study and Rebollo et al. In the Rebollo et al study, validity was tested at six months post-transplant, whereas the average time post-transplant in the current study was greater than 39 months, a period of time in which patients are likely to adjust to their clinical post-transplant status, which in turn is likely to influence their HQoL. ${ }^{18}$ Given that renal transplant recipients who are at least two years post-transplant are generally more clinically stable compared with renal transplant recipients within the first year post-transplant, we likewise believe our findings represent a more stable assessment of HQoL.

Like all studies, ours has limitations. The generalizability of the results may be limited by the sample size of this study and the demographic makeup of the sample. Female and non-white renal transplant recipients are somewhat overrepresented compared with the general US transplant population: female renal transplant recipients, $48 \%$ of the sample versus $40 \%$ of the US population; and non-white renal transplant recipients, $50 \%$ of the sample versus $41 \%$ of the US population. ${ }^{19}$ Another limitation is the lack of an objective measure of renal function or other physiological indicators of graft (transplanted kidney) function or rejection. We were thus unable to stratify patients by disease severity to test the validity of the KTQ further. However, since all renal transplant recipients were taking immunosuppressant therapy according to pharmacy refill records, during the time of instrument completion we assume that all study participants had functioning grafts. Possible ceiling and floor effects were evaluated and determined to be minimal for the SF-12v2, but the KTQ subscales (particularly the KTQ-appearance) had moderate to substantial ceiling effects. The substantial (84\%) ceiling effect indicated for the KTQ-appearance subscale may have contributed to the lack of significance associated with this measure and the PCS-12, but generally did not appear to influence the findings pertaining to other KTQ subscales. Despite these limitations, we believe the study results are indicative of good concurrent validity of the KTQ in our sample of US renal transplant recipients.

\section{Conclusion}

HQoL is an important outcome in the renal transplant population, and valid and reliable transplant-specific instruments are needed to conduct comprehensive assessment of HQoL. Thus, we examined and found considerable support for the concurrent validity of the KTQ, a HQoL instrument specific to renal transplantation in US renal transplant recipients. Future studies should continue exploring the validity of the KTQ, as well as its practical and research utility in HQoL measurement in the renal transplant population.

\section{Acknowledgment}

This publication was made possible by grant number 1R01DK081347-01A2 from the National Institute of Diabetes and Digestive and Kidney Diseases at the National Institutes of Health. Its contents are solely the responsibility of the authors and do not necessarily represent the official views of the sponsors.

\section{Disclosure}

The authors declare they have no conflicts of interests to report in this work.

\section{References}

1. The WHOQOL Group. The World Health Organization quality of life assessment (WHOQOL): position paper from the World Health Organization. Soc Sci Med. 1995;41:1403-1409.

2. Rebollo P, Ortega F, Ortega T, Valdés C, García-Mendoza M, Gómez E. Spanish validation of the "Kidney Transplant Questionnaire": a useful instrument for assessing health related quality of life in kidney transplant patients. Health Qual Life Outcomes. 2003;1:56.

3. Fiebiger W, Mitterbauer C, Oberbauer R. Health-related quality of life outcomes after kidney transplantation. Health Qual Life Outcomes. 2004;2:2.

4. Bohlke M, Marini SS, Rocha M, et al. Factors associated with health-related quality of life after successful kidney transplantation: a population-based study. Qual Life Res. 2009;18:1185-1193.

5. Laupacis A, Keown P, Pus N, et al. A study of the quality of life and cost-utility of renal transplantation. Kidney Int. 1996;50: 235-242.

6. Laupacis A, Pus N, Muirhead N, Wong C, Ferguson B, Keown P. Disease-specific questionnaire for patients with a renal transplant. Nephron. 1993;64:226-231.

7. Rostami Z, Tavallaii S-A, Jahani Y, Einollahi B. Assessment of quality of life in a single-center transplantation population using the Kidney Transplant Questionnaire-25 Questionnaire. Transplant Proc. 2011;43:590-591.

8. Eysenck MW. Psychology: An International Perspective. New York, NY: Psychology Press; 2004.

9. Monette DR, Sullivan TJ, DeJong CR. Applied Social Research: A Tool for the Human Services. Belmont, CA: Brooks/Cole; 2011.

10. Streiner DL, Norman GR. Health Measurement Scales: A Practical Guide to Their Development and Use. 4th ed. New York, NY: Oxford University Press; 2008.

11. Ware J, Kosinski M, Turner-Bowker D, Gandek B. User's Manual for the $S F-12 v 2^{\circledR}$ Health Survey (with a supplement documenting SF-12 ${ }^{\circledR}$ health survey). Lincoln, RI: QualityMetric Incorporated; 2009. 
12. Liem YS, Bosch JL, Arends LR, Heijenbrok-Kal MH, Hunink MG. Quality of life assessed with the Medical Outcomes Study Short Form 36-Item Health Survey of patients on renal replacement therapy: a systematic review and meta-analysis. Value Health. 2007:10:390-397.

13. Stavem K, Ganss R. Reliability and validity of the ESRD Symptom Checklist-Transplantation Module in Norwegian kidney transplant recipients. BMC Nephrol. 2006;7:17.

14. Barotfi S, Molnar MZ, Almasi C, et al. Validation of the Kidney Disease Quality of Life-Short Form questionnaire in kidney transplant patients. J Psychosom Research. 2006;60:495-504.

15. McHorney CA, Tarlov AR. Individual-patient monitoring in clinical practice: are available health status surveys adequate? Qual Life Res. $1995 ; 4: 293-307$.
16. George D, Mallery P. SPSS for Windows Step by Step: A Simple Guide and Reference. 11.0 Update. 4th ed. Boston, MA: Allyn and Bacon; 2003.

17. Sakthong P, Charoenvisuthiwongs R, Shabunthom R. A comparison of EQ-5D index scores using the UK, US, and Japan preference weights in a Thai sample with type 2 diabetes. Health Qual Life Outcomes. 2008;6:71.

18. Sprangers MAG, Schwartz CE. Integrating response shift into healthrelated quality of life research: a theoretical model. Soc Sci Med. 1999;48:1507-1515.

19. Organ Procurement Transplantation Network. National data. Available at: http://optn.transplant.hrsa.gov/latestData/step2.asp. Accessed January 31, 2011.

\section{Publish your work in this journal}

Patient Preference and Adherence is an international, peer-reviewed, open access journal focusing on the growing importance of patient preference and adherence throughout the therapeutic continuum. Patient satisfaction, acceptability, quality of life, compliance, persistence and their role in developing new therapeutic modalities and compounds to optimize clinical outcomes for existing disease states are major areas of interest. This journal has been accepted for indexing on PubMed Central. The manuscript management system is completely online and includes a very quick and fair peer-review system. Visit http://www.dovepress.com/ testimonials.php to read real quotes from published authors. 